\title{
AIRCRAFT DYNAMIC LATERAL STABILITY ANALYTIC FORMULATION FOR DUTCH ROLL MODE STABILITY PREDICTION
}

\author{
M. J. Bazile and M. F. Calderara
}

AIRBUS

Route de bayonne 316, Toulouse 3100, France

Currently, the civil aircraft manufacturers during the development have strong will to relax the natural aircraft stability, in order to optimize mainly performances in term of fuel consumption, by reducing lifting control surfaces, which is not without consequences on aircraft stability. The study of new innovating configurations, as the flying wing aircraft with the engines in rear position, requires a deepening of the predictive methods of static and dynamic stability linked with the new aerodynamic aspects. On the topic of dynamic stability, the first presentation was made with conference EUCASS 2007 in Brussels. This presentation focused on the longitudinal aspect that highlighted new analytical expressions of the manoeuvre point, likely to improve the prediction of the dynamic margin at the stage of future project studies. In the same spirit, this paper describes a similar work that investigated the study of lateral dynamic stability. This work studied dynamic lateral stability according to the center of gravity location and allowed to determine on the Dutch roll damping curve a point beyond which the lateral model is instable. The first part of this study presents considerations on Dutch roll damping mode leading to analytic resolution. The second part will propose examples of this method applied to $\mathrm{A} / \mathrm{C}$ design phase and perspectives of this method for new $\mathrm{A} / \mathrm{C}$ concepts.

\section{NOMENCLATURE}

$q \quad$ Pitch rate

$V \quad$ True air speed

$\alpha \quad$ Angle of attack

This is an Open Access article distributed under the terms of the Creative Commons Attribution-Noncommercial License 3.0, which permits unrestricted use, distribution, and reproduction in any noncommercial medium, provided the original work is properly cited. 


$$
\begin{array}{ll}
\eta_{i j}=\frac{2 I_{i, j}}{\rho S_{\mathrm{ref}} L_{\mathrm{ref}}^{3}} & \text { Reduced inertia with } i=j=x, y, z, \\
\mu=\frac{2 M}{\rho S_{\mathrm{ref}} L_{\mathrm{ref}}} & \text { Reduced mass } \\
\xi_{\mathrm{DR}} & \text { Dutch roll damping } \\
\omega_{\mathrm{DR}} & \text { Dutch roll frequency } \\
\omega_{R} & \text { Roll frequency } \\
\omega_{\mathrm{SRM}} & \text { Short period mode frequency }
\end{array}
$$

\section{INTRODUCTION}

This paper describes an analytic method for lateral aircraft behavior to calculate an equivalent point of the longitudinal "manoeuvre point." This point is presented as the limit of the dynamic stability of the aircraft coming from the Dutch roll oscillation analysis and can be found by considering the damping equal to zero: beyond this limit, the natural aircraft is dynamically instable. The position of this point along $\mathrm{A} / \mathrm{C}$ fuselage axis is strongly linked to the $\mathrm{A} / \mathrm{C}$ geometry and aerodynamic that means for classical A/C geometry this point is far on rear fuselage axis, but for new A/C configurations, this point may be in the flight domain. In this case, the interest of this analytic method is to immediately provide this limit instead of requiring many lateral state-space models function of the center of gravity location, for determining the particular Cog position that cancels the Dutch roll damping mode.

\section{REMIND OF SOME LONGITUDINAL CONSIDERATIONS ABOUT DYNAMIC STABILITY}

Even for longitudinal A/C sizing in classical flight domain, the phugoid mode is neglected because this period is much longer than the short period mode (SPM). With this hypothesis, the flight mechanic model obtained is said "the SPM model" as follows with an elevator input:

$$
\left\{\begin{array}{c}
\dot{\alpha} \\
\dot{q}
\end{array}\right\}=\left[\begin{array}{cc}
C_{\alpha}^{*} & C_{q}^{*} \\
D_{\alpha}^{*} & D_{q}^{*}
\end{array}\right]\left\{\begin{array}{c}
\alpha \\
q
\end{array}\right\}+\left[\begin{array}{c}
C_{\delta q}^{*} \\
D_{\delta q}^{*}
\end{array}\right] \delta q .
$$

Based on this model, the dynamic stability analysis (according to the current center of gravity location $X_{\mathrm{cg}}$ ) leads to solve the following equation:

$$
\omega_{\mathrm{SPM}}^{2}\left(X_{\mathrm{cg}}\right)=0
$$

where

$$
\omega_{\mathrm{SPM}}^{2}=\left(C_{\alpha}^{*} D_{q}^{*}-D_{\alpha}^{*} C_{q}^{*}\right) .
$$


It has been demonstrated [1] that the solution is given by the following expression:

$$
\frac{X_{\mathrm{MP}}}{L_{\mathrm{ref}}}=\frac{X_{\mathrm{cg}}}{L_{\mathrm{ref}}}-\frac{I_{y y}}{m V L_{\mathrm{ref}}} \frac{\omega_{\mathrm{SPM}}^{2}\left(X_{\mathrm{cg}}\right)}{C_{\alpha}^{*}} .
$$

This $X_{\mathrm{MP}}$ point represents the rear center of gravity position beyond which the $\mathrm{A} / \mathrm{C}$ is dynamically unstable. This point is also matching with the manoeuvre point according the definition found in [2] and defined as: The point where the elevator angle per " $g$ " is equal to zero. The mean idea of these few considerations is to identify this limit point on longitudinal short period mode model by solving

$$
\omega_{\mathrm{SPM}}^{2}\left(X_{\mathrm{cg}}\right)=0 .
$$

\section{LATERAL FLIGHT MECHANIC MODEL}

According to hypothesis [3] on sideslip and bank angles, the lateral state-space model can be expressed as follows with aileron and rudder input:

$$
\left\{\begin{array}{c}
\dot{\beta} \\
\dot{p} \\
\dot{r} \\
\dot{\phi}
\end{array}\right\}=\left[\begin{array}{cccc}
C_{\beta}^{*} & C_{p}^{*} & C_{r}^{*} & C_{\phi}^{*} \\
l_{\beta}^{*} & l_{p}^{*} & l_{r}^{*} & l_{\phi}^{*} \\
n_{\beta}^{*} & n_{p}^{*} & n_{r}^{*} & n_{\phi}^{*} \\
0 & 1 & \tan \theta_{0} & q_{0} \tan \theta_{0}
\end{array}\right]\left\{\begin{array}{l}
\beta \\
p \\
r \\
\phi
\end{array}\right\}+\left[\begin{array}{cc}
C_{\delta p}^{*} & C_{\delta r}^{*} \\
l_{\delta p}^{*} & l_{\delta r}^{*} \\
n_{\delta p}^{*} & n_{\delta r}^{*} \\
0 & 0
\end{array}\right]\left\{\begin{array}{l}
\delta p \\
\delta r
\end{array}\right\} .
$$

The eigen values analysis for classical flight domain leads to consider three modes:

(1) one real root (great in absolute value and near $l_{p}^{*}$ ), corresponding to the roll mode;

(2) one real root closed to zero, corresponding to the spiral mode; and

(3) two complex conjugated roots corresponding to Dutch roll mode.

In this conditions, the fourth order characteristic polynomial is given by the following expression:

$$
\Delta=\left(a P^{4}+b P^{3}+c P^{2}+d P+e\right) .
$$

One simple way to treat the lateral dynamic stability problematic is to consider the same hypothesis than longitudinal: The long period mode contribution is neglected for classical flight domain. It means that the following terms in state space $[A]$ matrix are cancelled:

$$
C_{\phi}^{*}=l_{\phi}^{*}=n_{\phi}^{*}=0 .
$$


In this condition, the initial fourth order characteristic polynomial becomes three order characteristic polynomial as follows:

$$
\Delta=P\left(a P^{3}+b P^{2}+c P+d\right) .
$$

In this particular case, the study shows that the point defining the dynamic limit lateral stability is given by the solution of the following expression:

$$
d\left(X_{\mathrm{cg}}\right)=0
$$

or by considering the $d$ coefficient as the product of the Dutch-roll mode frequency square by the roll mode frequency as follows:

$$
\omega_{\mathrm{DR}}^{2}\left(X_{\mathrm{cg}}\right)=\frac{d\left(X_{\mathrm{cg}}\right)}{\omega_{R}\left(X_{\mathrm{cg}}\right)}=0 .
$$

It can be demonstrated by considering the definition of the manoeuvre point - adapted to lateral behavior - that this point is the manoeuvre point whatever the lateral model behavior and will be noted $\left(X_{\mathrm{MP}} / L_{\mathrm{ref}}\right)$. To sum up these considerations, the initial hypothesis leads to consider and to solve the same parameter than for longitudinal case to obtain the limit stability point; consequently, the dynamic stability criteria is given by the Dutch-roll frequency.

Now, the question is: If this restrictive hypothesis given by (1) is cancelled, how becomes the dynamic instability?

In fact, when this hypothesis is cancelled, the dynamic stability criteria is given by the Dutch-roll damping equal to zero; so,

$$
\xi_{\mathrm{DR}}\left(X_{\mathrm{cg}}\right)=0 .
$$

The solution is noted $\left(X_{\mathrm{MP}} / L_{\mathrm{ref}}\right)^{*}$. In fact, the simplification (1) leading to $\left(X_{\mathrm{MP}} / L_{\mathrm{ref}}\right)$ could be a great approximation if this point, whatever the flight and mass condition, could be located at more forward position than the

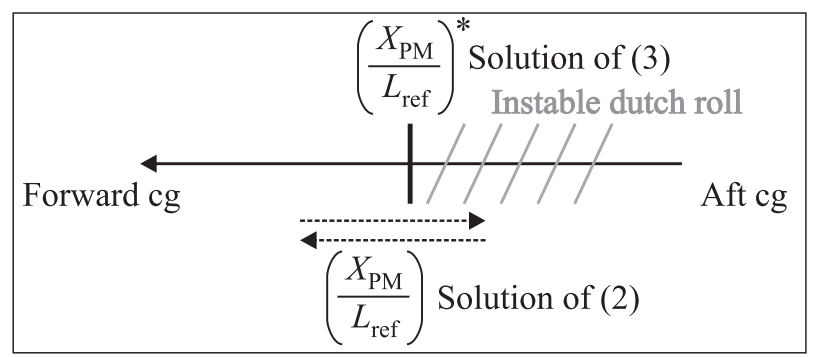

Figure 1 On demonstration of identification of the stability limit point 
point $\left(X_{\mathrm{MP}} / L_{\mathrm{ref}}\right)^{*}$. If one were sure of this property, it will be sufficient to identify $\left(X_{\mathrm{MP}} / L_{\mathrm{ref}}\right)$ "to cover" the real limit of stability $\left(X_{\mathrm{MP}} / L_{\mathrm{ref}}\right)^{*}$ (Fig. 1$)$.

But calculations show that it is not the case. The $\left(X_{\mathrm{MP}} / L_{\mathrm{ref}}\right)^{*}$ point be located at more forward position than $\left(X_{\mathrm{MP}} / L_{\mathrm{ref}}\right)$ for particular set of mass, inertia, dynamic pressure, and Mach number. This result means the $\omega_{\mathrm{DR}}^{2}\left(X_{\mathrm{cg}}\right)$ $=0$ criteria is not a robust one, and the only way to identify the stability limit point in flight domain is to solve the reminded Eq. (3).

Based on this result, the following section proposes to develop the method to solve the upper expression.

\section{LATERAL STABILITY CRITERIA}

It has been said in the previous section that the only robust criteria for lateral dynamic stability analyse was to solve expression (3). To achieve this goal, the analytic solution of the fourth order characteristic polynomial must be developed. It means that the Dutch-roll frequency and damping mode analytic expressions must be identified by considering in this case $C_{\phi}^{*} \neq 0$ but $\theta_{0}=q_{0}=l_{\phi}^{*}=n_{\phi}^{*}=0$ in a first time. These two expressions are founded by using the Cardan-Ferrari method. The Ferrari's method allows to transform the fourth order characteristic polynomial into a three-order characteristic polynomial and the Cardan's methods allow to find by introducing intermediate coefficients for the exact solution, and consequently, the exact frequency and damping analytic expression of the Dutch-roll mode, and the two others, of course. At this stage, it can be demonstrated that the constraint (3) implies directly the following relation noted $\bar{\omega}$ between the four coefficients contained in the fourth-order polynomial expression:

$$
\bar{\omega}=b^{2} e+d^{2}-b c d .
$$

So, based on relation (4), it is possible to find $\left(X_{\mathrm{MP}} / L_{\mathrm{ref}}\right)^{*}$ leading to (3) constraint that implies $\bar{\omega}\left(\left(X_{\mathrm{MP}} / L_{\mathrm{ref}}\right)^{*}\right)=0$, too. For example, Fig. 2 plots the Dutch-roll damping mode evolution for constant angle of attack and the $\bar{\omega}$ function according to the center of gravity location.

Figure 2 shows the two functions behavior and the crossing point $\left(X_{\mathrm{MP}} / L_{\mathrm{ref}}\right)^{*}$ (circle) that is to be predicted with an analytical process. One can also see: if $\bar{\omega}\left(X_{\mathrm{cg}}\right)<0$, the lateral model is stable, and if $\bar{\omega}\left(X_{\mathrm{cg}}\right)>0$ - unstable.

The next step considering the $\bar{\omega}$ expression is to replace the four coefficients $b$, $c, d$, and $e$ expressed function of the $\Delta X$ parameter as $\Delta X=X_{M} / L_{\mathrm{ref}}-X_{\mathrm{cg}} / L_{\mathrm{ref}}$ function of the aerodynamic data reference $X_{\mathrm{cg}} / L_{\mathrm{ref}}$. After many transformations, expressions for the coefficients $b, c, d$, and $e$ are as follows:

- coefficient $b=-\left(C_{\beta}^{*}+n_{r}^{*}+l_{p}^{*}\right)$ :

$$
b(\Delta X)=b\left(X_{\mathrm{cg}}\right)+b_{1} \Delta X+b_{2} \Delta X^{2} ;
$$




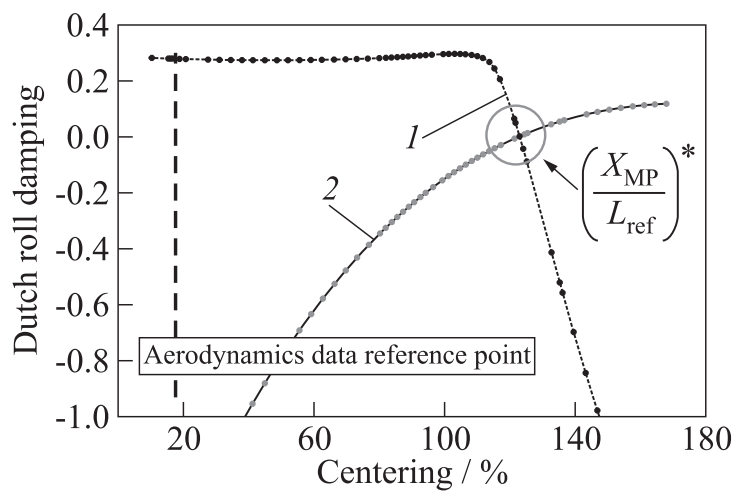

Figure 2 Ditch-roll damping mode evolution for constant angle of attack (1) and the $\bar{\omega}$ function (2) according to the center of gravity location

- coefficient $c=\left(C_{\beta}^{*} l_{p}^{*}-C_{p}^{*} l_{\beta}^{*}\right)+\left(C_{\beta}^{*} n_{r}^{*}-C_{r}^{*} n_{\beta}^{*}\right)+\left(n_{r}^{*} l_{p}^{*}-n_{p}^{*} l_{r}^{*}\right)$ :

$$
c(\Delta X)=c\left(X_{\mathrm{cg}}\right)+c_{1} \Delta X+c_{2} \Delta X^{2}
$$

- coefficient $d=\left(C_{p}^{*}\left(l_{\beta}^{*} n_{r}^{*}-n_{\beta}^{*} l_{r}^{*}\right)+l_{p}^{*}\left(n_{\beta}^{*} C_{r}^{*}-C_{\beta}^{*} n_{r}^{*}\right)+n_{p}^{*}\left(C_{\beta}^{*} l_{r}^{*}-l_{\beta}^{*} C_{r}^{*}\right)\right)$ $-C_{\phi}^{*} l_{\beta}^{*}$ :

$$
d(\Delta X)=d\left(X_{\mathrm{cg}}\right)+d_{1} \Delta X
$$

- coefficient $e=C_{\phi}^{*}\left(n_{r}^{*} l_{\beta}^{*}-l_{r}^{*} n_{\beta}^{*}\right)$ :

$$
e(\Delta X)=e\left(X_{\mathrm{cg}}\right)+e_{1} \Delta X
$$

where coefficients $b_{1}, b_{2}, c_{1}, c_{2}, d_{1}$, and $e_{1}$ depend only on the aerodynamic data at the reference point and the longitudinal equilibrium data toward the angle of attack $\alpha_{\text {eq }}$. For example, coefficient $b_{1}$ is given by

$$
\begin{aligned}
b_{1}=-\left(\mu \frac { V } { L _ { \mathrm { ref } } } \frac { 1 } { \eta _ { x x } \eta _ { z z } - \eta _ { z x } \eta _ { x z } } \left(\eta_{x z}\left(C_{p}^{*}-\sin \alpha_{\mathrm{eq}}\right)+\eta_{x x}\left(C_{r}^{*}+\right.\right.\right. & \left.\left.\cos \alpha_{\mathrm{eq}}\right)\right) \\
& \left.+n_{\beta}^{*} \frac{L_{\mathrm{ref}}}{V}\right) .
\end{aligned}
$$

Based on expressions (5)-(8) and substituting them into Eq. (4), one finally obtains a five-order polynomial expression that allows to determine the $\left(X_{\mathrm{MP}} / L_{\mathrm{ref}}\right)^{*}$ abscissa:

$$
\bar{\omega}(\Delta X)=A_{5} \Delta X^{5}+A_{4} \Delta X^{4}+A_{3} \Delta X^{3}+A_{2} \Delta X^{2}+A_{1} \Delta X+A_{0} .
$$


The basic idea was that with the first simplified hypothesis, it was possible to find an explicit analytical expression of the limit stability point as a function of mass, inertia, and flight point conditions in the same way that the longitudinal manoeuvre point [1]. With this accurate proposed method, it is clearly impossible because the $\left(X_{\mathrm{MP}} / L_{\mathrm{ref}}\right)^{*}$ abscissa is the result of a five-order polynomial calculation.

The numerical resolution leads to three real positive or negative roots and two complex conjugated roots. With experience, the physical solution is given by the root closed to the unit, but in a global process, one has to pay attention to capture the good solution by putting in place a numerical treatment.

Whatever Eq. (9) allows to determine precisely the point $\left(X_{\mathrm{MP}} / L_{\mathrm{ref}}\right)^{*}$ leading to $\xi\left(\left(X_{\mathrm{MP}} / L_{\mathrm{ref}}\right)^{*}\right)=0$.

It means for a given flight point and mass characteristics that the proposed method predicts the limit center of gravity position beyond which the lateral model is dynamically unstable. This point abscissa is a function of flight point mass characteristics and $\mathrm{A} / \mathrm{C}$ aerodynamics as shows the following relation:

$$
\begin{array}{r}
\left(\frac{X_{\mathrm{MP}}}{L_{\mathrm{ref}}}\right)^{*}=f\left(\rho, V, M, I_{x x}, I_{z z}, I_{x z}, \alpha_{\mathrm{eq}},\right. \text { stationary } \\
\text { and instationary laterial aerodynamics coefficient }) .
\end{array}
$$

Most of time, for classical A/C configurations, this point is far on rear fuselage and the Aircraft lateral stability is not a real problematic. The most limiting aft $\mathrm{cg}$ is linked to longitudinal instability. To illustrate this theorical part let show an example with $\alpha_{\text {eq }}=0$ :

$$
\begin{array}{ll}
\text { Mach number: } & \mathrm{M}=0.45 \\
\text { True air speed: } & V=152.93 \mathrm{~m} / \mathrm{s} \\
\text { Altitude: } & H=0 \mathrm{ft} \\
\text { Center of gravity: } & X_{\mathrm{cg}}=20.94 \% \\
\text { Mass: } & M=120 \mathrm{t} \\
\text { Inertia: } & I_{x x}=50 \mathrm{~m}, I_{z z}=90 \mathrm{~m}, I_{x z}=8 \mathrm{~m}
\end{array}
$$

The five roots are as follows: $S_{1}=20.9378 ; S_{2}=3.2703+2.7187 i ; S_{3}$ $=3.2703-2.7187 i ; S_{4}=1.7953 ;$ and $S_{5}=1.0813$.

The physical solution is given by $S_{5}$, it leads to $\left(X_{\mathrm{MP}}\right)^{*}=118.2145 \%$ (mass.). This example clearly shows for classical A/C configuration with limit point beyond the classical A/C flight domain. This numerical calculation has been proposed to validate the method. This value should not be kept in mind. 


\section{USE OF THE METHOD FOR A/C DESIGN PHASE}

In the last section, an innovative method is proposed to predict the limit point of lateral A/C stability. So, for lateral and longitudinal aerodynamic states of the $\mathrm{A} / \mathrm{C}$ associated to a center of gravity, it is possible to locate $\left(X_{\mathrm{MP}} / L_{\mathrm{ref}}\right)^{*}$. At this stage, two ways are possible:

(1) if there are no available data to determine the longitudinal equilibrium, $\left(X_{\mathrm{MP}} / L_{\mathrm{ref}}\right)^{*}$ can be identified with $\alpha_{\mathrm{eq}}=0$. This first hypothesis allows to have an idea of its position; and

(2) if the longitudinal aerodynamics data are known, the first simplification consist in calculating the equilibrium at aerodynamic reference point to provide $\alpha_{\text {eq }}$ and after that to consider this value constant along the cog variation. In this case, the limit point $\left(X_{\mathrm{MP}} / L_{\mathrm{ref}}\right)^{*}$ is given by Eq. (9).

The last step is to improve the last process by considering for each variation of center of gravity to the $\alpha_{\text {eq }}$ is calculated and introduced in Eq. (9) to have the $\left(X_{\mathrm{MP}} / L_{\mathrm{ref}}\right)^{*}$ value. After several values of $\operatorname{cog}$, there exists one unique value noted $\left(X_{\mathrm{MP}} / L_{\mathrm{ref}}\right)^{*}$ that verifies both $\xi\left(\left(X_{\mathrm{MP}} / L_{\mathrm{ref}}\right)^{* *}\right)=0$ and the longitudinal equilibrium in forces and in moment given by the following system:

$$
\begin{aligned}
C z_{\alpha}\left(\alpha_{\mathrm{eq}}-\alpha_{0}\right)+C z_{i h} i h_{\mathrm{eq}} & =\mu\left(\frac{g L_{\mathrm{ref}}}{V^{2}}\right) ; \\
C m_{0}+C m_{\alpha}\left(\alpha_{\mathrm{eq}}-\alpha_{0}\right)+C m_{i h} i h_{\mathrm{eq}}+C m_{\text {engines }} & =0 .
\end{aligned}
$$

This last process is a little bit more complex than the second one but leads to an accurate limit stability point calculation. Furthermore, for a rapid prediction, the second way is more appropriate. At last, to have an idea of the relative point location, the following part proposes numerical values with $\mathrm{A} / \mathrm{C}$ data used in section 3 :

- with $\alpha_{\text {eq }}=0$,

$$
\left(\frac{X_{\mathrm{MP}}}{L_{\mathrm{ref}}}\right)^{*}=118.2145 \% \text { (mass.) }
$$

- with $\alpha_{\text {eq }}$ value at the reference point $\left(X_{\text {eq }} / L_{\text {ref }}\right)$,

$$
\left(\frac{X_{\mathrm{MP}}}{L_{\mathrm{ref}}}\right)^{*}=128.89 \% \text { (mass.) ; }
$$

- with $\alpha_{\text {eq }}$ varying with $\operatorname{cog}\left(X_{\mathrm{cg}} / L_{\mathrm{ref}}\right)$,

$$
\left(\frac{X_{\mathrm{MP}}}{L_{\mathrm{ref}}}\right)^{* *}=126.16 \% \text { (mass.) } .
$$


For this set of data, a slight difference is observed between the second and the third approaches.

The method has been illustrated with an example leading to $\left(X_{\mathrm{MP}} / L_{\mathrm{ref}}\right)^{*}$ values out of the classical flight domain. Theses values for classical A/C configuration are unusual and must be considered as a mean to validate the methodology. Whatever this method must be understood as an innovative method for new A/C concept to predict, in the same way that in longitudinal, the stability limit point.

Concerning this methodology applied for the lateral sizing activities on classical A/C geometry, most of time, the engine failure is considered as a design criteria for the vertical taiplane, because engines are located under the wing with a great $y$ axis level arm in regard of fuselage; so, if the vertical taiplane is well sized, this surface must be able to counter the yaw moment induced by this most critical engine failure case. For future concept, it may be possible that the engine is located on rear fuselage near axis. In this condition, the engine failure case will not be probably an appropriate criterion to size the VTP. In this condition, the more appropriate design criteria for the VTP could be the Dutch roll stability and in this perspective, the knowledge of the $\left(X_{\mathrm{MP}} / L_{\mathrm{ref}}\right)^{*}$ point defining the stability limit point could be necessary. This possible new A/C could lead to an "relaxed lateral stability" concept.

\section{CONCLUDING REMARKS}

This paper presents an analytic method to predict the stability limit point for lateral model beyond which the model is dynamically unstable. This methodology can be used in $\mathrm{A} / \mathrm{C}$ predevelopment phase.

This methodology can be applied to A/C development to verify if, in the flight domain, the A/C lateral stability for mass cases several with a great time reduction calculation. This methodology can be applied to $\mathrm{A} / \mathrm{C}$ design phase to predict this limit stability point vs. geometric parameters according to three possible ways as focused in section 4 .

This paper has proposed this methodology with some restrictive hypothesis into lateral state-space matrix as $\theta_{0}=q_{0}=l_{\phi}^{*}=n_{\phi}^{*}=0, I_{x z}$ inertia independent of the center of gravity location. The limit stability point in open loop was also considered. The next work to improve the limit stability point calculation is to take into account $\theta_{0}, q_{0}, l_{\phi}^{*}, n_{\phi}^{*}$, parameters engine aerodynamic, and $I_{x z}$ as a function of $\operatorname{cog}$ in open loop. This first study allows putting in place the method core, and based on this, the suit consists to take into account the second-order effect.

Then, the next step is to be able to predict the limit stability point in closed loop by considering a simple feedback gain on rudder and aileron as $\delta r=K_{r} r$ and 
$\delta p=K_{r} p$. This simple feedback model allows to keep fourth-order characteristic polynomial. In case of adding a time delay to the model, the rudder transfer function in low frequencies, the $\bar{\omega}$ criteria will not be available because a fifthorder characteristic polynomial will be obtained.

\section{REFERENCES}

1. Bazile, J. New analytic formulation of the manoeuvre point of a flexible aircraft for the dynamic stability analysis. 2nd European Conference for Aerospace Sciences.

2. De Castro, H. V. 2001. The longitudinal static stability of tailless aircraft. COA Report No. 0018/1.

3. Wanner, J.-C. Dynamique du vol. Sup'aéro. 